\title{
MỐI QUAN HỆ GIŨ๋A DANH TIẾNG, TRÁCH NHIỆM XÃ HộI DOANH NGHIÊP, NIỀM TIN VÀ Ý ĐINH MUA HÀNG CỦA KHÁCH HÀNG TRONG NGÀNH Ô TÔ VIẸTT NAM
}

\author{
NGUYỄN NGỌC HIỀN \\ Trường Đại học Công nghiệp thành phố Hồ Chí Minh - Phân hiệu Quảng Ngãi; \\ nguyenngochien.qn@iuh.edu.vn
}

Tóm tắt. Nghiên cứu đề xuất và kiểm định tác động của danh tiếng đến nhận thức trách nhiệm xã hội doanh nghiệp và ý định mua hàng của khách hàng, trong đó niềm tin đóng vai trò trung gian và ý định mua hàng được xem là kết quả cuối cùng. Các mối quan hệ này được kiểm định thông qua 1.002 mẫu khách hàng sở hữu và sử dụng ô tô du lịch tại Việt Nam. Kết quả nghiên cứu cho thấy, danh tiếng có tác động trực tiếp đến nhận thức trách nhiệm xã hội doanh nghiệp của khách hàng, đồng thời tác động gián tiếp thông qua trung gian là niềm tin. Ngoài ra, niềm tin và nhận thức trách nhiệm xã hội doanh nghiệp của khách hàng tác động đến ý định mua hàng. Tuy nhiên, danh tiếng không có tác động đến ý định mua hàng của khách hàng.

Từ khóa. Danh tiếng; Trách nhiệm xã hội doanh nghiệp; Niềm tin; Ý định mua hàng.

\section{THE RELATIONSHIP BETWEEN CORPORATE REPUTATION, CORPORATE SOCIAL RESPONSIBILITY, TRUST AND CUSTOMER PURCHASE INTENTION IN AUTOMOTIVE INDUSTRY IN VIET NAM}

\begin{abstract}
This study proposes and tests the impact of reputation on perceived corporate social responsibility, purchase intention of customers as well as the mediating role of customer trust, whereas purchase intention regarded as the final result. By using the sample of 1,002 customers, who owned and used passenger cars in Viet Nam, the results show that reputation has a direct impact on consumer perception of corporate social responsibility and the effect is found to partially mediate through customer trust. In addition, customer trust and consumer perception of corporate social responsibility affect the purchase intention. However, reputation has no impact on purchase intention of people.
\end{abstract}

Keywords. Reputation; Corporate social responsibility; Trust; Purchase intention.

\section{GIỚI THIỆU}

Trong môi trường kinh doanh đầy biến động và mức độ cạnh tranh cao như hiện nay, các doanh nghiệp luôn tìm kiếm cách để khác biệt hóa sản phẩm và xây dựng mối quan hệ thuận lợi với các bên liên quan. Nghiên cứu luôn cho thấy rằng danh tiếng là tài sản hiếm, khó có thể bắt chước, có giá trị và có quan hệ nhân quả không rõ ràng [1]. Do tầm quan trọng của danh tiếng như một tài sản vô hình có giá trị mà các doanh nghiệp phải quản lý một cách cẩn thận, hiểu biết được các yếu tố tiềm năng có thể tăng cường danh tiếng cũng như kết quả của danh tiếng có tầm quan trọng chiến lược [5].

Một trong những yếu tố có mối quan hệ với danh tiếng đã được nghiên cứu trong học thuật là trách nhiệm xã hội doanh nghiệp. Tuy nhiên, giữa trách nhiệm xã hội doanh nghiệp và danh tiếng có mối quan hệ nhân quả không rõ ràng. Pérez (2015) [26] cho rằng mối quan hệ giữa trách nhiệm xã hội doanh nghiệp và danh tiếng có thể là mối quan hệ hai chiều, trong đó trách nhiệm xã hội doanh nghiệp không chỉ ảnh hưởng đến danh tiếng mà ngược lại, danh tiếng cũng có thể ảnh hưởng đến nhận thức trách nhiệm xã hội doanh nghiệp của khách hàng. Ngoài ra, vấn đề này vẫn còn tương đối mới trong bối cảnh các nước đang phát triển [8].

Niềm tin và ý định mua hàng của khách hàng được xem như là kết quả của danh tiếng [1]. Trong khi ý định mua hàng của khách hàng được xem xét như là kết quả cuối cùng bởi nó đại diện cho hành vi thực tế [2], thì bản chất về mối quan hệ giữa danh tiếng và niềm tin vẫn còn mơ hồ. Một số tác giả cho rằng niềm tin được xem đơn thuần như là một trong những thuộc tính hoặc là tiền đề để đo lường danh tiếng 


\section{MỐI QUAN HỆ GIỮA DANH TIÊNG, TRÁCH NHIỆM XÃ HỘI DOANH NGHIÊP, NIỀM TIN VÀ Ý ĐỊNH MUA HÀNG CỦA KHÁCH HÀNG TRONG NGÀNH Ô TÔ VIỆT NAM}

[29]. Trong khi đó, một số nghiên cứu xem xét niềm tin như là kết quả của danh tiếng [18]. Vì vậy việc xem xét kiểm định lại mối quan hệ này là cần thiết.

Từ những thông tin làm nền tảng trên, nghiên cứu này nhằm giải thích cơ chế tác động giữa danh tiếng, trách nhiệm xã hội doanh nghiệp, niềm tin và ý định mua hàng của khách hàng. Cụ thể, niềm tin và trách nhiệm xã hội doanh nghiệp được xem là kết quả của danh tiếng, trong đó niềm tin được xem xét như biến trung gian trong mối quan hệ giữa danh tiếng và trách nhiệm xã hội doanh nghiệp. Ý định mua hàng được xem là kết quả cuối cùng.

\section{CƠ SỞ LÝ THUYẾT VÀ KHUNG PHÂN TÍCH}

\subsection{Cơ sở lí thuyết và giả thuyết nghiên cứu}

\subsubsection{Danh tiếng (Corporate Reputation)}

Khái niệm danh tiếng đã được quan tâm lớn của các nhà nghiên cứu từ những năm 1950. Tuy nhiên, có sự nhầm lẫn và thay thế cho nhau giữa các khái niệm danh tiếng, bản sắc và hình ảnh doanh nghiệp [38]. Fombrun và Van Riel (1997) [11] xác định bản sắc như là sự nhận thức của các nhà quản lý và người lao động (các bên liên quan nội bộ) về một doanh nghiệp, trong khi hình ảnh được xác định như là nhận thức được tổ chức bởi các bên liên quan bên ngoài. Danh tiếng do đó sẽ là sự kết hợp của hình ảnh và bản sắc trong một đại diện tập thể. Weiss và cộng sự (1999) [39] lập luận rằng hình ảnh mang tính "nội bộ" nhiều hơn, trong đó nó đại diện cho "bức tranh" dự định mà doanh nghiệp muốn công chúng nhìn thấy. Danh tiếng được phát triển từ sự giải thích được thực hiện bởi công chúng dựa trên các tín hiệu nhận được.

Walker (2010) [38] quan sát thấy rằng ba thuộc tính quan trọng được gán cho danh tiếng dựa trên định nghĩa của Fombrun (1996) [10]: (1) dựa trên nhận thức; (2) là nhận thức tổng hợp của tất cả các bên liên quan; và (3) là so sánh. Danh tiếng là "Tương đối ổn định, tổng hợp vấn đề cụ thể đại diện nhận thức của các hành động quá khứ của một doanh nghiệp và triển vọng trong tương lai khi so sánh với một số tiêu chuẩn" [38] (Trang 370 ).

\subsubsection{Trách nhiệm xã hội doanh nghiệp (CSR - Corporate Social Responsibility)}

Lý thuyết các bên liên quan cho rằng doanh nghiệp không nên chỉ xem xét lợi ích của cổ đông mà là lợi ích của tất cả các bên liên quan. Bên liên quan được xác định là bất kỳ nhóm hoặc cá nhân nào mà có thể ảnh hưởng hoặc bị ảnh hưởng bởi việc đạt được các mục tiêu của tổ chức [12]. Cách tiếp cận đa chiều sử dụng rộng rãi nhất được đề xuất bởi Carroll (1979) [7]. Ông đề xuất rằng một khái niệm đầy đủ của CSR nên bao gồm các thành phần kinh tế, pháp lý, đạo đức và từ thiện. Nghĩa vụ trong thành phần kinh tế bao gồm như tối đa hóa lợi nhuận cho các cổ đông, thiết lập một vị thế cạnh tranh mạnh mẽ, duy trì một mức độ cao về hiệu quả hoạt động, và tất cả những điều này là vì chỉ có khả năng sinh lời thì ba thành phần khác của CSR mới có thể được xây dựng. Nghiên cứu này xem xét CSR dụa trên nhận thức của khách hàng về các hoạt động CSR nhu thế nào, tù đó ảnh hưởng đến thái độ và hành vi của khách hàng.

\subsubsection{Niềm tin (Trust)}

Trong bối cảnh tiếp thị, niềm tin xuất hiện khi một bên trong một giao dịch thương mại có sự tin cậy ở một số tính năng cụ thể của bên kia. Từ một góc độ tâm lý, chức năng niềm tin là để giảm lo âu của khách hàng trong tình huống khó xử trong quá trình ra quyết định, dẫn đển giảm chi phí giao dịch do tìm kiếm và kiểm tra thông tin [20]. Niềm tin có thể cung cấp cảm giác an toàn những gì sẽ giúp tổ chức sống sót trong môi trường phức tạp. Từ quan điểm của người tiêu dùng, niềm tin đã được hiểu như sự tin tưởng của người tiêu dùng rằng doanh nghiệp sẽ thực hiện một cách phù hợp với mong đợi của họ [25]. Như vậy, niềm tin là sự tin tưởng vào một tổ chức sẽ không hành động gây hại, hoạt động đáng tin cậy và hoạt động theo hướng có lợi cho cả hai bên. Niềm tin được xem là cầu nối giữa kinh nghiệm trong quá khứ và dự đoán hành động trong tương lai.

\subsection{4. Ý định mua hàng}

Ý định mua hàng đại diện cho khả năng mà người tiêu dùng sẽ có kế hoạch hoặc sẵn sàng để mua một sản phẩm hoặc dịch vụ nhất định trong tương lai. Xem xét ý định mua hàng là điều cần thiết cho mỗi doanh nghiệp, bởi vì doanh nghiệp sẽ nổ lực để thực hiện và dành ngân sách cho việc xúc tiến và phát 


\section{MỐI QUAN HỆ GIỮA DANH TIÊNG, TRÁCH NHIỆM XÃ Hô̂I DOANH NGHIỆP, NIỀM TIN VÀ Ý ĐỊNH MUA HÀNG CỦA KHÁCH HÀNG TRONG NGÀNH Ô TÔ VIỆT NAM}

triển sản phẩm, các doanh nghiệp mong đợi khách hàng của mình quay trở lại [3]. Y định mua hàng có thể được sử dụng như là một chỉ số để dự đoán hành vi tiêu dùng của khách hàng, đại diện cho ý thức chủ quan hoặc khả năng mua hàng của khách hàng [9].

\subsubsection{Mối quan hệ giũa danh tiếng và trách nhiệm xã hội của doanh nghiệp}

CSR được sử dụng tốt nhất trong điều kiện danh tiếng tốt và không có khủng hoảng, trong khi danh tiếng xấu có thể dẫn đến cảm nhận đạo đức giả suy ra từ các động cơ bên trong của hoạt động CSR [33]. Pérez (2015) [26] cho rằng mối quan hệ giữa nhận thức trách nhiệm xã hội doanh nghiệp và danh tiếng có thể là mối quan hệ hai chiều, trong đó nhận thức trách nhiệm xã hội không chỉ ảnh hưởng đến danh tiếng mà ngược lại danh tiếng cũng có thể ảnh hưởng đến nhận thức trách nhiệm xã hội doanh nghiệp của khách hàng. Dựa trên những phân tích có thể thấy rằng, danh tiếng có ảnh hưởng đến nhận thức hành vi đạo đức của doanh nghiệp. Các hoạt động CSR được đánh giá cao nếu công ty có danh tiếng tốt, và được xem là hoạt động tư lợi nếu công ty có danh tiếng xấu. Và giả thuyết được đưa ra như sau:

\section{$H_{1}$ : Danh tiếng có tác động cùng chiều đến trách nhiệm xã hội doanh nghiệp.}

\subsubsection{Mối quan hệ giũa danh tiếng doanh nghiệp và niềm tin của khách hàng}

Các tài liệu hiện nay cho thấy một khoảng trống trong khái niệm cấu trúc niềm tin - đó là những gì định hướng và các thành phần của nó $[17,14]$. Van Der Merwe và Puth (2014) [37] dựa vào lý thuyết hệ thống xã hội học để giải thích mối quan hệ giữa danh tiếng và niềm tin. Nghiên cứu cho rằng, sự thành công của một tổ chức dựa vào cách quản lý mối quan hệ với các bên liên quan. Mối quan hệ giữa một tổ chức và các bên liên quan được xác định bởi sự không chắc chắn và rủi ro do phụ thuộc lẫn nhau [23]. Niềm tin được xem như là chìa khóa chiến lược có thể được sử dụng để làm giảm bớt sự không chắc chắn, cải thiện hiệu quả và quản lý mối quan hệ của một tổ chức với các bên liên quan của nó. Van Der Merwe và Puth (2014) [37] đã đề xuất khung khái niệm trong đó danh tiếng được xem như tiền đề của niềm tin. Dựa trên quan điểm lý thuyết này, giả thuyết được đưa ra như sau:

\section{$\mathrm{H}_{2}$ : Danh tiếng có tác động cùng chiều đến niềm tin của khách hàng}

\subsubsection{Mối quan hệ giũa niềm tin và trách nhiệm xã hội của doanh nghiệp}

Khách hàng có một thái độ và niềm tin tích cực đối với các doanh nghiệp tham gia các hoạt động CSR [28]. Tuy nhiên, vẫn chưa có nghiên cứu tác động của niềm tin đối với nhận thức trách nhiệm xã hội doanh nghiệp. Khi khách hàng có niềm tin đối với một doanh nghiệp, họ sẽ cho rằng doanh nghiệp đó tuân thủ các nguyên tắc đạo đức, quan tâm đến phúc lợi xã hội [24], dẫn đến nhận thức tích cực về CSR. Dựa trên quan điểm này, giả thuyết được đưa ra như sau:

$H_{3}$ : Niềm tin của khách hàng có tác động cùng chiều đến trách nhiệm xã hội doanh nghiệp.

\subsubsection{Mối quan hệ giũa danh tiếng và ý định mua hàng}

Helm (2005) [16] cho rằng đánh giá của các bên liên quan dựa trên kinh nghiệm với các nhà sản xuất đã tác động tích cực đến danh tiếng và lòng trung thành. Do đó, việc phát triển danh tiếng ở mức độ cao là quan trọng để đảm bảo khả năng cạnh tranh và tạo ra hành vi tích cực. Brown (1998) [6] đã tìm thấy danh tiếng có ảnh hưởng tích cực đến thái độ của người mua đối với sản phẩm, ý định mua hàng và hành vi mua thực tế. Có ý kiến rằng danh tiếng tốt có thể dẫn đến những kết quả có lợi được sự đồng ý của. Vì vậy, không có nghi ngờ rằng danh tiếng có thể đóng góp vào sự phản hồi thuận lợi từ các bên liên quan đổi với doanh nghiệp. Giả thuyết được đưa ra như sau:

$H_{4}$ : Danh tiếng có tác động cùng chiều đến ý định mua hàng.

\subsubsection{Mối quan hệ giũa trách nhiệm xã hội doanh nghiệp và ý định mua hàng}

Những sáng kiến CSR dẫn đến thái độ tích cực hơn đối với các doanh nghiệp, cũng như sự gia tăng ý định mua của khách hàng, nhưng chỉ khi họ nhận thức được sáng kiến cụ thể [32]. Nghiên cứu ở Mỹ cho thấy CSR trở thành yếu tố mà rất có thể làm cho khách hàng trung thành với một thương hiệu hay một doanh nghiệp cụ thể [22]. Đánh giá từ các nghiên cứu trước đây cho thấy rằng, CSR tác động không rõ ràng đến ý định mua hàng của khách hàng. Tuy nhiên, có bằng chứng cho thấy CSR tạo ra kết quả tích cực cho kinh doanh của các doanh nghiệp. Nó cũng được đề nghị về mặt lý thuyết và thực nghiệm rằng hành vi trách nhiệm xã hội của doanh nghiệp có tiềm năng ảnh hưởng đến quyết định mua hàng. Vì vậy, có thể đưa ra giả thuyết rằng: 


\section{MỐI QUAN HỆ GIỮA DANH TIÊNG, TRÁCH NHIỆM XÃ HỘI DOANH NGHIÊP, NIỀM TIN VÀ Ý ĐỊNH MUA HÀNG CỦA KHÁCH HÀNG TRONG NGÀNH Ô TÔ VIỆT NAM}

H5: Trách nhiệm xã hội doanh nghiệp có tác động cùng chiều đến ý định mua hàng của khách hàng.

\subsubsection{Mối quan hệ giũa niềm tin và ý định mua hàng của khách hàng}

Bart và cộng sự (2005) [4] tìm thấy một mối quan hệ mạnh mẽ giữa niềm tin và ý định hành vi. Khuynh hướng hành vi có thể bao gồm sự sẳn sàng để thực hiện các hoạt động tiếp theo, chẳng hạn như mua lại sản phẩm từ doanh nghiệp. Niềm tin dựa trên kinh nghiệm trước đó đóng một vai trò rất quan trọ̣ng trong việc thúc đẩy ý định mua của người tiêu dùng. Hơn nữa, niềm tin ảnh hưởng đến sự sẵn sàng để mua từ một doanh nghiệp cụ thể [15]. Từ những tranh luận trên, có thể đưa ra giả thuyết:

$H_{6}$ : Niềm tin có tác động cùng chiều đến ý định mua hàng của khách hàng

\subsubsection{Niềm tin của khách hàng nhu là biến trung gian}

Các hoạt động CSR được đánh giá cao nếu doanh nghiệp có danh tiếng tốt và được xem là hoạt động tư lợi nếu có danh tiếng xấu [40]. Ngoài ra, các doanh nghiệp có danh tiếng tốt được dự kiến sẽ cư xử tốt và ít tham gia vào các hành vi tiêu cực, nên tăng cường niềm tin của khách hàng. Đồng thời, khi khách hàng có niềm tin đối với doanh nghiệp, họ sẽ cho rằng doanh nghiệp đó tuân thủ các nguyên tắc đạo đức, quan tâm đến phúc lợi xã hội [24] và dẫn đến nhận thức tích cực về CSR. Dựa trên quan điểm này, giả thuyết được đưa ra như sau:

$H_{7}$ : Niềm tin của khách hàng là trung gian tác động của danh tiếng đến trách nhiệm xã hội doanh nghiẹp.

\subsection{Mô hình nghiên cứu}

Danh tiếng được biết đến như là tín hiệu thông tin về các hoạt động trong quá khứ và tương lai của các doanh nghiệp, ảnh hưởng đến cách thức khách hàng nhìn nhận và đối xử với doanh nghiệp [10]. Trong nghiên cứu này, lý thuyết tín hiệu dùng để giải thích tác động của danh tiếng đến CSR, niềm tin và ý định mua hàng của khách hàng. Ngoài ra, dựa vào "khung giá trị nhận thức - Valence Frameword" là quan điểm bổ sung của lý thuyết hành động hợp lí được đề xuất bởi Fishbein và Ajzen (1975) [9], Kim và cộng sự (2009) [19] đã đề xuất khung nhận thức mở rộng, trong đó niềm tin ảnh hưởng trực tiếp đến ý định mua hàng, đồng thời niềm tin cũng ảnh hưởng gián tiếp thông qua hai trung gian là nhận thức lợi ích và nhận thức rủi ro. Lí thuyết này dùng để giải thích mối quan hệ giữa niềm tin, CSR và ý định mua hàng của khách hàng.

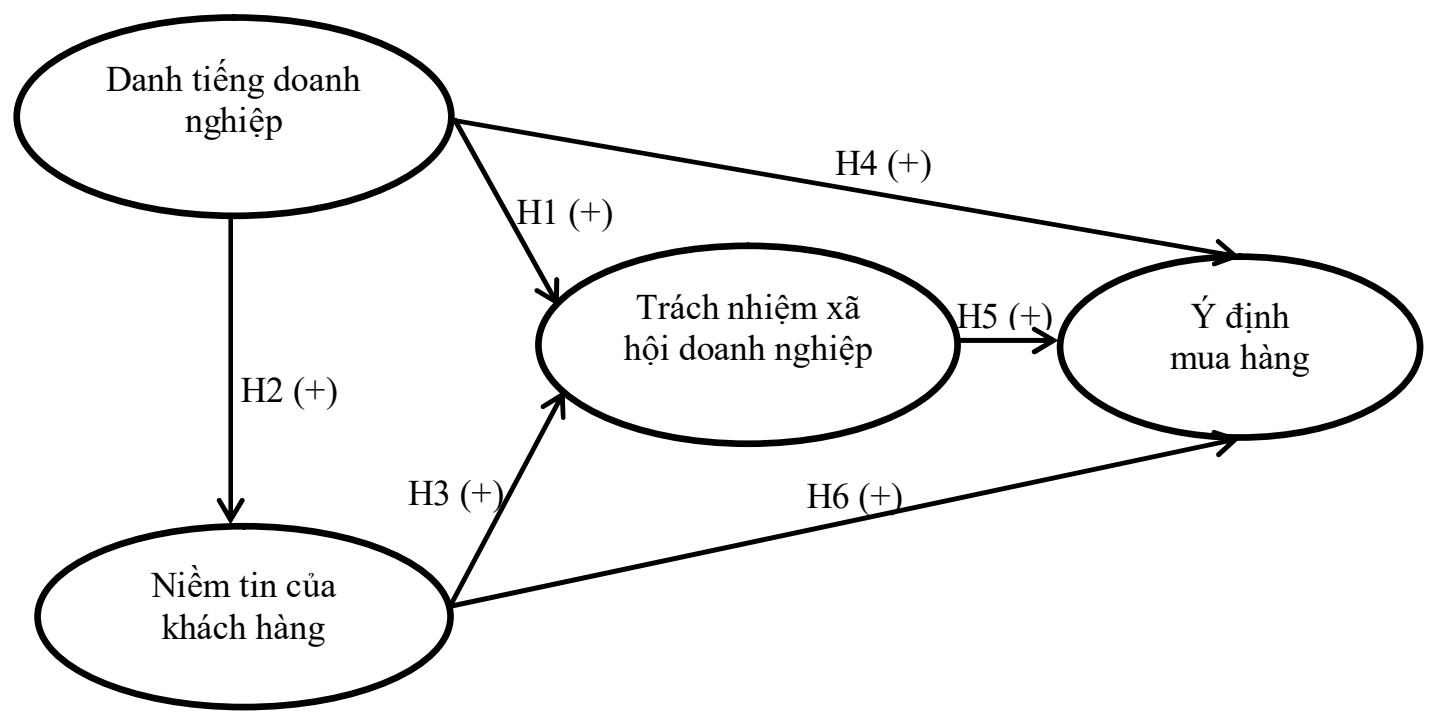

$\mathrm{H} 7$

Hinh 1. Mô hình nghiên cứu đề xuất Nguồn: Tác giả đề xuất 
MỐI QUAN HÊ GIỮA DANH TIÊNG, TRÁCH NHIỆM XÃ HỘI DOANH NGHIÊP, NIỀM TIN VÀ Ý ĐỊNH MUA HÀNG CỦA KHÁCH HÀNG TRONG NGÀNH Ô TÔ VIỆT NAM

\section{PHƯƠNG PHÁP NGHIÊN CÚU}

Nghiên cứu sử dụng kết hợp phương pháp nghiên cứu định tính và phương pháp nghiên cứu định lượng. Nghiên cứu định tính được sử dụng để điều chỉnh thang đo phù hợp với điều kiện cụ thể tại Việt Nam và nghiên cứu định lượng được sử dụng để kiểm định các giả thuyết trong mô hình nghiên cứu.

\section{1. Đo lường}

Nhận thức về CSR được phát triển từ thang đo của Singh và cộng sự (2008) [34]. Thang đo này được lựa chọn vì đo lường dựa trên nhận thức của khách hàng và bao gồm tất cả các khía cạnh theo cách tiếp cận đa chiều. Thang đo danh tiếng được phát triển từ thang đo lường RepTrak ${ }^{\mathrm{TM}}$ Pulse của Ponzi và cộng sự (2011) [29]. Do có nhiều quan điểm đo lường khác nhau và các khái niệm này mới tại Việt Nam. Vì vậy, ngoài việc tham khảo các nghiên cứu trước, tác giả đã tiến hành thảo luận nhóm tập trung ( 8 khách hàng sở hữu và sử dụng xe ô tô du lịch). Kết quả cho thấy, hầu hết các biến được xem xét tương tự như thang đo đã lựa chọn, đồng thời họ cho rằng thang đo CSR nên bổ sung một số biến đo lường trong thành phần trách nhiệm thương mại bao gồm: "Luôn nổ lực cải thiện sản phẩm và dịch vụ cho khách hàng", "Thông báo chính xác và trung thực về đặc điểm sản phẩm" và "Doanh nghiệp trung thực trong hoạt động quảng cáo/khuyến mãi”. Ngoài ra, Danh tiếng là sự tích lũy nhận thức của công chúng. Vì vậy, họ cho rằng nên bổ sung đánh giá của công chúng về doanh nghiệp dựa trên nhận thức của người trả lời. Kết quả một thang đo lường gồm ba thành phần: trách nhiệm thương mại, trách nhiệm đạo đức, trách nhiệm xã hội và 14 biến, thang đo lường danh tiểng gồm 5 biến. Các thang đo được xây dựng dựa trên thang đo lường Singh và cộng sự (2008) [34], Sandhu và Kapoor (2010) [30], Ponzi và cộng sự (2011) [29] và từ thảo luận nhóm.

Thang đo của Sirdeshmukh và cộng sự (2002) [35], Selnes và Sallis (2003) [31] được sử dụng để đo lường niềm tin của khách hàng. Ý định mua hàng được đo lường bằng thang đo đã được chấp nhận từ các nghiên cứu Sirohi và cộng sự (1998) [36], Lam và cộng sự (2004) [21].

\subsection{Thu thập dữ liệu}

Phỏng vấn trực tiếp được thực hiện tại gara rửa xe ô tô, siêu thị, bãi đỗ xe... tại TP.HCM, Quảng Ngãi và Đà Nẵng. Đối tượng khảo sát là những khách hàng cá nhân sở hữu và sử dụng xe ô tô du lịch, nhằm khảo sát thị trường tương đối đồng nhất. Nghiên cứu chính thức được thực hiện từ tháng 15/02/2017 đến 20/04/2017. Tổng số phiếu điều tra là 1.200 phiếu, tổng số phiếu thu về là 1.079 phiếu. Trong quá trình nhập dữ liệu, số phiếu không đạt yêu cầu là 77 phiếu (Chủ yếu là không điền đầy đủ và chỉ điền một ý kiến). Số phiếu hợp lệ là 1.002 phiếu được chọn làm dữ liệu nghiên cứu chính thức cho nghiên cứu này.

\section{KẾT QUẢ VÀ THẢO LUẬN}

\subsection{Kết quả nghiên cứu}

\subsection{1. Đặc điểm mẫu thống kê}

Người trả lời có đặc điểm chủ yếu là: có giới tính là nam, có độ tuổi tập trung từ 26 đến 55 tuổi, có trình độ phổ biến từ trung học phổ thông đến sau đại học và có thu nhập từ 10 triệu đến trên 25 triệu. Thương hiệu xe ô tô du lịch được khảo sát nhiều nhất là Toyota, nước xuất xứ thương hiệu được khảo sát nhiều nhất là Nhật Bản và Hàn Quốc, xe ô tô được sản xuất, lắp ráp trong nước chiếm tỷ lệ cao nhất. Như vậy, mẫu thống kê phù hợp với thị phần hiện tại của các thương hiệu xe ô tô tại Việt Nam.

\subsubsection{Kiểm định thang đo}

\subsubsection{1. Đánh giá độ tin cậy thang đo}

Hệ số Cronbach alpha được sử dụng rộng rãi nhất để đo lường độ tin cậy của thang đo. Cronbach alpha lớn hơn 0,6 [13]. Ngoài ra, hệ số tương quan biến tổng lớn hơn hoặc bằng 0,3 thì biến đó đạt yêu cầu [27]. Kết quả đánh giá độ tin cậy thang đo như sau: 


\section{NIỀM TIN VÀ Ý ĐỊ̂HH MUA HÀNG CỦA KHÁCH HÀNG TRONG NGÀNH Ô TÔ VIỆT NAM}

Bảng 1. Độ tin cậy của thang đo

\begin{tabular}{|c|c|c|c|c|c|}
\hline TT & Thang đo thành phần & $\begin{array}{c}\text { Số biến } \\
\text { quan sát }\end{array}$ & $\begin{array}{c}\text { Tương quan } \\
\text { biến tồng }\end{array}$ & $\begin{array}{c}\text { Cronbach's } \\
\text { Alpha }\end{array}$ & Ghi chú \\
\hline 1 & Danh tiếng & 5 & $\geq 0,642$ & 0,867 & \\
\hline 2 & Trách nhiệm thương mại & 5 & $\geq 0,703$ & 0,893 & $\begin{array}{c}\text { Loại biến } \\
\text { TNTM4 }\end{array}$ \\
\hline 3 & Trách nhiệm đạo đức & 3 & $\geq 0,681$ & 0,846 & $\begin{array}{c}\text { Loại biến } \\
\text { TNDD1 }\end{array}$ \\
\hline 4 & Trách nhiệm xã hội & 4 & $\geq 0,592$ & 0,822 & \\
\hline 5 & Niềm tin của khách hàng & 5 & $\geq 0,647$ & 0,880 & \\
\hline 6 & Ý định mua hàng & 3 & $\geq 0,793$ & 0,902 & \\
\hline
\end{tabular}

(Nguồn: Kết quả xủ lý số liệu trên phần mềm SPSS 22)

Bảng 2. Trọng số nhân tố của thang đo

\begin{tabular}{|l|c|}
\hline \multicolumn{1}{|c|}{ Khái niệm và biến quan sát } & Trọng số \\
nhân tố
\end{tabular}

(Nguồn: Kết quả xủ lý số liệu trên phần mềm SPSS 22.0) 


\section{MỐI QUAN HỆ GIỬA DANH TIẾNG, TRÁCH NHIẸM XÃ HỘI DOANH NGHIỆ, NIỀM TIN VÀ Ý ĐỊNH MUA HÀNG CỦA KHÁCH HÀNG TRONG NGÀNH Ô TÔ VIỆT NAM}

Kết quả kiểm định trong bảng 1 cho thấy, tất cả thang đo thành phần đều có hệ số Cronbach alpha lớn hơn 0,6 , đảm bảo độ tin cậy cần thiết. Tuy nhiên, biến TNTM4 có hệ số tương quan biến tổng là 0,249 nhỏ hơn 0,3 và biến TNDD1 có hệ số tương quan biến tổng là 0,279 nhỏ hơn 0,3 nên loại các biến này. Kết quả kiểm định sau khi loại biến cho thấy các biến đều đạt độ tin cậy cần thiết.

\subsubsection{2. Đánh giá giá trị của thang đo - phân tích nhân tố khám phá (EFA)}

Những thang đo đã được đánh giá độ tin cậy sẽ được đưa vào đánh giá giá trị thang đo bằng cách phân tích nhân tố khám phá (EFA). Phép trích nhân tố Principal Axis Factoring (PAF) và phép quay không vuông góc Promax được sử dụng. Kết quả phân tích EFA cho thấy, hệ số $\mathrm{KMO}=0,903>0,5$ và $\operatorname{Sig}($ Kiểm định Bartlett $)=0,00<0,05$, cho thây phân tích EFA là phù hợp.

Bảng 2 cho thấy các biến trong mô hình nghiên cứu được trích thành 6 nhân tố có phương sai trích là $70,889 \%>50 \%$. Trọng số nhân tố của các biến đo lường đều lớn hơn 0,5 . Chênh lệch giữa các trọng số lớn hơn 0,3 . Như vậy, các biến đều đạt giá trị hội tụ.

\subsubsection{Phân tích nhân tố khẳng định (CFA)}

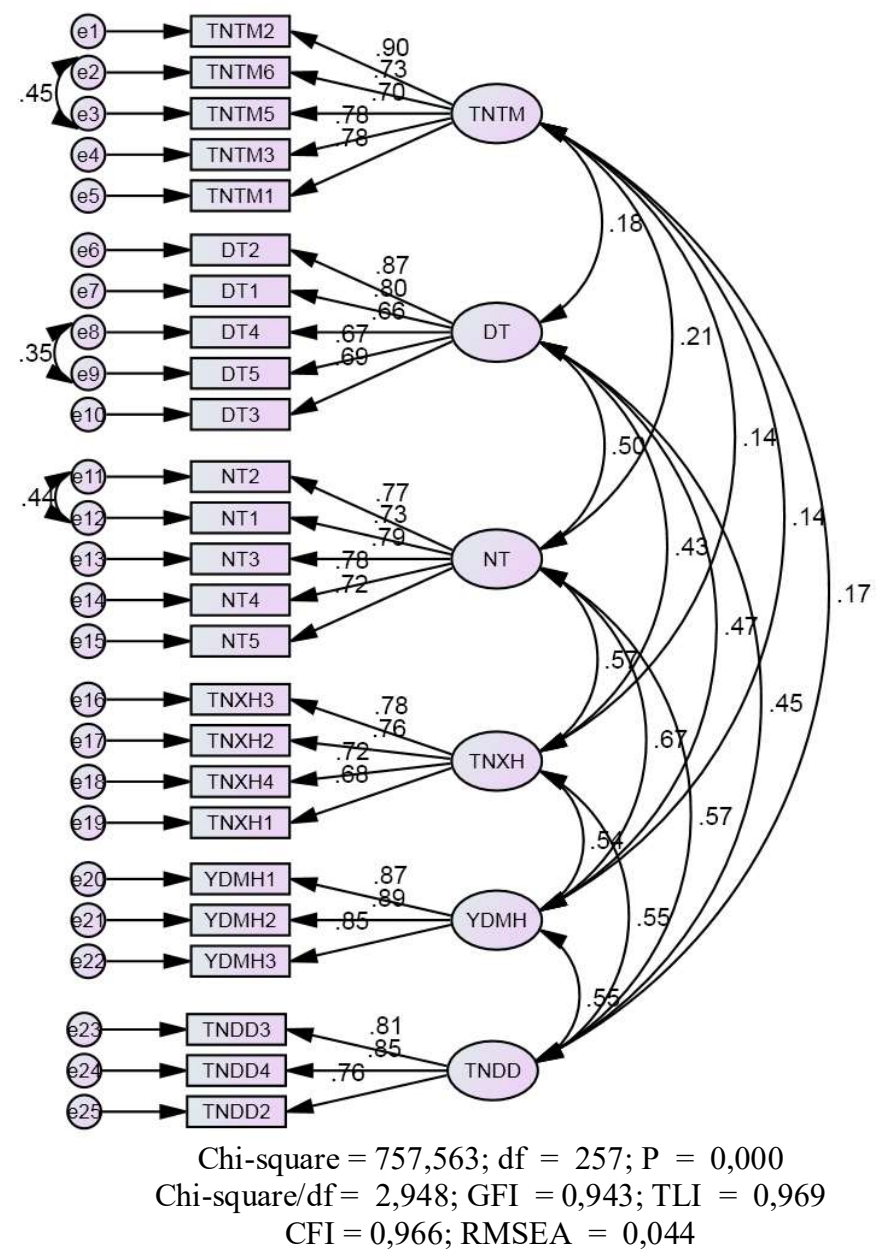

Hình 2. Mô hình CFA tới hạn (chuẩn hóa)

Mô hình tới hạn được sử dụng để đánh giá tất cả các khái niệm trong mô hình. Kết quả cho thấy có 257 bậc tự do và mô hình này thích hợp với dữ liệu thị trường (Chi-square/df $=2,95<3 ; \mathrm{CFI}=0,996>$ $0,9 ; \mathrm{GFI}=0,943>0,9 ; \mathrm{TLI}=0,960>0,9$ và $\mathrm{RMSEA}=0,044<0,08)$. Các thang đo trách nhiệm xã hội, ý định mua hàng, trách nhiệm đạo đức không có tương quan giữa các sai số đo lường nên các biến quan sát đạt được tính đơn hướng, các thang đo trách nhiệm thương mại, danh tiếng và niềm tin của khách 


\section{MỐI QUAN HỆ GIỬA DANH TIẾNG, TRÁCH NHIẸM XÃ HỘI DOANH NGHIỆ, NIỀM TIN VÀ Ý ĐỊNH MUA HÀNG CỦA KHÁCH HÀNG TRONG NGÀNH Ô TÔ VIỆT NAM}

hàng không đạt tính đơn hướng. Các trọng số chuẩn hóa của các biến quan sát giao động từ $0,60-0,90$ đều đạt yêu cầu (lớn hơn 0,5$)$ và các trọng số chưa chuẩn hóa đều có ý nghĩa thống kề $(\mathrm{p}=0,00)$ với độ tin cậy $95 \%$, nên các biến quan sát dùng để đo lường các khái niệm đạt giá trị hội tụ. Ngoài ra, hệ số tương quan của từng cặp khái niệm khác biệt so với 1 có ý nghĩa thống kê nên các thành phần đạt giá trị phân biệt (Hình 2).

Kết quả kiểm định độ tin cậy và phương sai trích của các khái niệm cho thấy, độ tin cậy Cronbach alpha và độ tin cậy tổng hợp của các thành phần đều lớn hơn 0,6 và phương sai trích lớn hơn 0,5 . Như vậy các thang đo đều đạt độ tin cậy cao.

\subsection{3. Ước lượng các mối quan hệ cấu trúc}

\subsubsection{Kiểm định mô hình lí thuyết bằng SEM}

Kết quả kiểm định mô hình cấu trúc được trình bày ở hình 3 cho thấy mô hình có 263 bậc tự do với $\mathrm{P}$-value $=0,00<0,05$. Các chỉ số đều phù hợp với dữ liệu thị trường (Chi-quare $/ \mathrm{df}<3 ; \mathrm{CFI}, \mathrm{GFI}, \mathrm{TLI}>$ $0,9$, RMSEA $<0,8)$.

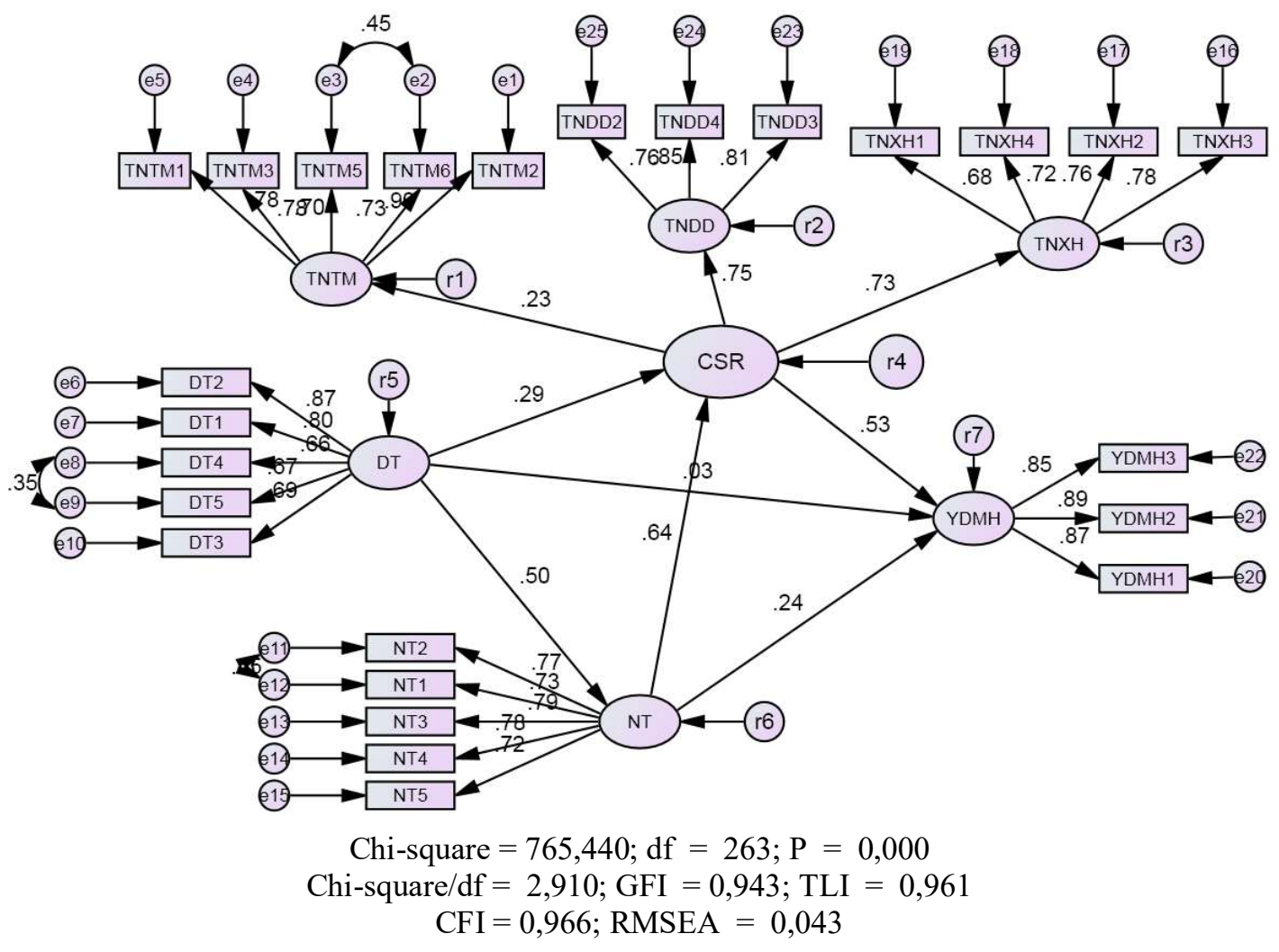

Hinh 3. Kết quả phân tích SEM mô hình lí thuyết (đã chuẩn hóa)

Kết quả ước lượng các tham số chính của mô hình được trình bày trong bảng 3 . Kết quả cho thấy các mối quan hệ trong mô hình đều có ý nghĩa thống kê vì có giá trị $\mathrm{p}<0,05$, ngoại trừ mối quan hệ giữa Danh tiếng và ý định mua hàng của khách hàng $(\mathrm{p}=0,462>0,05)$. Như vậy, các giả thuyết $\mathrm{H}_{1}, \mathrm{H}_{2}, \mathrm{H}_{3}$, $\mathrm{H}_{5}, \mathrm{H}_{6}$ được chấp nhận, giả thuyết $\mathrm{H}_{4}$ bị bác bỏ. 
Bảng 3. Hệ số hồi quy các mối quan hệ trong mô hình lý thuyết

\begin{tabular}{|c|c|c|c|c|c|c|}
\hline $\begin{array}{l}\text { Giả } \\
\text { thuyết }\end{array}$ & Mối quan hệ & $\begin{array}{l}\text { Hệ số chưa } \\
\text { chuẩn hóa }\end{array}$ & $\begin{array}{c}\text { Hể số } \\
\text { chuẩn hóa }\end{array}$ & SE & CR & P-value \\
\hline H1 & $\begin{array}{l}\text { Danh tiếng } \rightarrow \text { Trách nhiệm xã } \\
\text { hội doanh nghiệp }\end{array}$ & 0,218 & 0,228 & 0,032 & 6,889 & 0,00 \\
\hline $\mathrm{H} 2$ & Danh tiếng $\rightarrow$ Niềm tin & 0,475 & 0,497 & 0,033 & 13,836 & 0,00 \\
\hline $\mathrm{H} 3$ & $\begin{array}{l}\text { Niềm tin } \rightarrow \text { Trách nhiệm xã } \\
\text { hội doanh nghiệp }\end{array}$ & 0,523 & 0,635 & 0,041 & 12,636 & 0,00 \\
\hline $\mathrm{H} 4$ & $\begin{array}{c}\text { Danh tiếng } \rightarrow \text { Ý định mua } \\
\text { hàng }\end{array}$ & 0,042 & 0,031 & 0,057 & 0,735 & 0,462 \\
\hline H5 & $\begin{array}{c}\text { Trách nhiệm xã hội doanh } \\
\text { nghiệp } \rightarrow \text { Ý định mua hàng }\end{array}$ & 0,933 & 0,530 & 0,166 & 5,621 & 0,00 \\
\hline H6 & Niềm tin $\rightarrow$ Ý định mua hàng & 0,349 & 0,241 & 0,101 & 3,461 & 0,00 \\
\hline
\end{tabular}

(Nguồn: Kết quả xủ̉ lý số liệu trên phần mềm SPSS 22)

\subsubsection{Phân tích trung gian}

Nghiên cứu vai trò trung gian của niềm tin trong mối quan hệ giữa danh tiếng và CSR là mục tiêu quan trợng của nghiên cứu. Để xác định tác động trung gian có ý nghĩa, bốn điều kiện cần đáp ứng: (1) danh tiểng tác động có ý nghĩa đến niềm tin; (2) Niềm tin tác động có ý nghĩa đến CSR; (3) danh tiếng có tác động có ý nghĩa đến CSR; và (4) Tác động của danh tiếng đến CSR không có ý nghĩa thống kê hoặc giảm khi tính toán tác động của biến trung gian (Andrews và cộng sự, 2004).

Hai điều kiện đầu tiên được kiểm tra bằng ước lượng mô hình 1 (Bảng 4). Kết quả cho thấy mô hình phù hợp với dữ liệu thị trường (Chi-square/df $=2,995 ; \mathrm{CFI}=0,967 ; \mathrm{GFI}=0,949 ; \mathrm{TLI}=0,962$ và

RMSEA $=0,044)$, các mối quan hệ trong mô hình đều có ý nghĩa thống kê vì có $\mathrm{P}$-value $<0,05$, nên điều kiện 1 và 2 được đáp ứng.

Điều kiện 3 được kiểm tra trong mô hình 2 (bảng 4), kết quả cho thấy mối quan hệ trong mô hình đều có ý nghĩa thống kê vì có $\mathrm{P}$-value $<0,05$. Nên điều kiện 3 được đáp ứng.

Cuối cùng, mô hình 3 (bảng 4) kiểm định tác động của danh tiếng đến CSR khi có biến trung gian niềm tin. Kết quả cho thấy mô hình phù hợp với dữ liệu thị trường $($ Chi-square $/ \mathrm{df}=2,761 ; \mathrm{CFI}=0,971$; $\mathrm{GFI}=0,951$; TLI $=0,966$ và $\mathrm{RMSEA}=0,041)$, các mối quan hệ trong mô hình đều có ý nghĩa thống kê vì có $\mathrm{P}$-value $<0,05$. Đồng thời, so sánh giữa mô hình 2 và mô hình 3 (Bảng 4 ) cho thấy danh tiếng tác động đến CSR giảm xuống khi có tác động của niềm tin (hệ số chuẩn hóa giảm từ 0,453 xuống 0,219 ), điều kiện 4 được đáp ứng.

Bảng 4. Các mô hình ước lượng mối quan hệ

\begin{tabular}{|c|c|c|c|c|c|}
\hline Mối quan hệ & $\begin{array}{c}\text { Hệ số chưa chuẩn } \\
\text { hóa }\end{array}$ & $\begin{array}{c}\text { Hệ số } \\
\text { chuẩn hóa }\end{array}$ & SE & $\mathbf{C R}$ & P-value \\
\hline \multicolumn{6}{|l|}{ Mô hình 1: Điều kiện 1 và 2} \\
\hline Danh tiếng $\rightarrow$ Niềm tin & 0,488 & 0,525 & 0,033 & 14,698 & 0,00 \\
\hline $\begin{array}{c}\text { Niềm tin } \rightarrow \text { Trách nhiệm xã } \\
\text { hội doanh nghiệp }\end{array}$ & 0,663 & 0,800 & 0,043 & 15,363 & 0,00 \\
\hline \multicolumn{6}{|c|}{ Kiểm định mô hình 2: Điều kiện 3} \\
\hline $\begin{array}{c}\text { Danh tiếng } \rightarrow \text { Trách nhiệm } \\
\text { xã hội doanh nghiệp }\end{array}$ & 0,453 & 0,602 & 0,038 & 12,015 & 0,00 \\
\hline \multicolumn{6}{|c|}{ Kiểm định mô hình 3: Điều kiện 4} \\
\hline Danh tiếng $\rightarrow$ Niềm tin & 0,459 & 0,497 & 0,033 & 13,873 & 0,00 \\
\hline $\begin{array}{l}\text { Niềm tin } \rightarrow \text { Trách nhiệm xã } \\
\text { hội doanh nghiệp }\end{array}$ & 0,524 & 0,636 & 0,042 & 12,548 & 0,00 \\
\hline $\begin{array}{c}\text { Danh tiếng } \rightarrow \text { Trách nhiệm } \\
\text { xã hội doanh nghiệp }\end{array}$ & 0,219 & 0,288 & 0,032 & 6,868 & 0,00 \\
\hline
\end{tabular}

(Nguồn: Kết quả xủ̉ lý số liệu trên phần mềm SPSS 22.0) 
Như vậy, ngoài tác động trực tiếp của danh tiếng đến CSR, niềm tin được xem như biến trung gian cho tác động gián tiếp của danh tiếng đến CSR (Giả thuyết 7 được chấp nhận).

\subsubsection{Kiểm định độ tin cậy ước lượng bằng Bootstrap}

Nghiên cứu này sử dụng phương pháp Bootstrap với số lượng mẫu lặp lại $\mathrm{N}=500$. Kết quả cho thấy trị tuyệt đối CR nhỏ so với 2 nên có thể nói là độ lệch nhỏ, không có ý nghĩa thống kê ở độ tin cậy 95\%. Như vậy, có thể kết luận ước lượng trong mô hình đáng tin cậy.

\subsection{Thảo luận kết quả nghiên cứu}

Kết quả kiểm định thang đo cho thấy, thang đo CSR là thang đo bậc 2 được đo lường bởi 3 thành phần (trách nhiệm thương mại, trách nhiệm đạo đức và trách nhiệm xã hội). Các thang đo danh tiếng, niềm tin và ý định mua hàng là thang đo lường bậc 1 và có ý nghĩa thống kê tại thị trường Việt Nam. Tất cả các thang đo được đo lường dựa trên nhận thức của khách hàng.

Về mối quan hệ giữa các khái niệm, danh tiếng tác động trực tiếp cùng chiều đến CSR, khi khách hàng đánh giá danh tiếng tốt họ sẽ cảm nhận các hoạt động CSR theo chiều hướng tích cực. Kết quả này phù hợp với nghiên cứu của Yoon và cộng sự (2006) cho rằng khách hàng có xu hướng nhận thức động cơ hoạt động từ thiện là chân thành, đáng tin cậy khi họ đánh giá tích cực về doanh nghiệp. Kết quả cũng chỉ ra niềm tin của khách hàng là trung gian một phần tác động của danh tiếng đến CSR. Như vậy, ngoài việc danh tiếng tác động trực tiếp đến CSR, danh tiếng sẽ tác động gián tiếp một phần thông qua niềm tin của khách hàng, khi doanh nghiệp có danh tiếng tốt sẽ dẫn đến khách hàng tin tưởng doanh nghiệp và từ niềm tin sẽ dẫn đến khách hàng cảm nhận tốt về các hoạt động CSR.

Nghiên cứu cũng cho thấy, CSR và niềm tin tác động tích cực đến ý định mua hàng, khi khách hàng nhận thức hoạt động CSR tích cực và có niềm tin vào doanh nghiệp thì ý định mua hàng của họ tăng lên. Tuy nhiên, danh tiếng không tác động trực tiếp đến ý định mua hàng, kết quả này trái ngược với nghiên cứu của Brown (1998). Kết quả này đã được tiến hành thảo luận sâu với 5 khách hàng sử dụng ô tô du lịch, kết quả cho thấy khách hàng đồng ý với kết quả này bởi vì ý định mua lại của khách hàng trong ngành ô tô phụ thuộc vào nhiều yếu tố (như giá cả, hiệu suất sử dụng, trải nghiệm sản phẩm mới...), còn danh tiếng sẽ tác động mạnh hơn khi có ý định mua lần đầu.

Xét về mức độ tác động, niềm tin tác động mạnh nhất đến trách nhiệm xã hội doanh nghiệp ( $\beta$ chuẩn hóa là 0,635 ), tiếp theo là mối quan hệ giữa trách nhiệm xã hội doanh nghiệp và ý định mua hàng của khách hàng $(\beta$ chuẩn hóa là 0,530$)$, tiểp theo là danh tiếng tác động đến niềm tin và niềm tin tác động đến ý định mua hàng ( $\beta$ chuẩn hóa lần lượt là 0,497 và 0,241$)$, và cuối cùng là tác động của danh tiếng đển trách nhiệm xã hội doanh nghiệp.

\section{KẾT LUẬN VÀ HÀM Ý QUẢN TRI}

\subsection{Kết luận}

Dựa vào các nghiên cứu trước đây, tác giả đã xây dựng mô hình mối quan hệ giữa danh tiếng, niềm tin, CSR và ý định mua hàng. Kết quả cho thấy sau khi điều chỉnh từ ngữ và bổ sung một số biến quan sát thông qua thảo luận nhóm, các thang đo lường CSR, danh tiếng, niềm tin và ý định mua hàng đạt độ tin cậy cao. Thứ hai, danh tiếng tác động trực tiếp đến CSR, ngoài ra niềm tin có vai trò trung gian trong mối quan hệ giữa danh tiếng và $\mathrm{CSR}$. Cuối cùng, $\mathrm{CSR}$ và niềm tin có tác động tích cực đến ý định mua hàng của khách hàng.

\subsection{Hàm ý quản trị}

Các nghiên cứu trước đây cho rằng sáng kiến trách nhiệm xã hội doanh nghiệp bao gồm 4 thành phần (trách nhiệm kinh tế, trách nhiệm pháp lí, trách nhiệm đạo đức, trách nhiệm từ thiện) (Carroll và Buchholtz, 2011). Tuy nhiên khi kiểm định thang đo dự trên nhận thức của khách hàng trong ngành ô tô tại thị trường Việt Nam, thang đo lường CSR có ba thành phần: trách nhiệm thương mại, trách nhiệm đạo đức và trách nhiệm xã hội. Như vậy, để nâng cao nhận thức của khách hàng về hoạt động CSR, các doanh nghiệp có thương hiệu ô tô tại Việt Nam cần tập trung vào trách nhiệm thương mại với khách hàng (Ví dụ: Duy trì chất lượng tốt, trung thực với khách hàng...), đồng thời các doanh nghiệp tập trung vào thực hiện kinh doanh đúng pháp luật và hỗ trợ các hoạt động mang tính chất xã hội. 

NIỀM TIN VÀ Ý ĐỊNH MUA HÀNG CỦA KHÁCH HÀNG TRONG NGÀNH Ô TÔ VIỆT NAM

Các nghiên cứu trước cho rằng CSR sẽ tác động tích cực đến danh tiếng (Fombrun và cộng sự, 2015). Nghiên cứu này phát hiện mối quan hệ ngược, đó là danh tiếng sẽ tác động trực tiếp đến CSR đồng thời tác động gián tiếp thông qua niềm tin của khách hàng. Khi một doanh nghiệp có danh tiếng tốt, điều này một phần sẽ làm cho khách hàng tin tưởng và đánh giá các hoạt CSR theo chiều hướng tốt, mặt khác tác động trực tiếp đến nhận thức CSR của khách hàng. Như vậy, để khách hàng đánh giá các hoạt động CSR theo chiều hướng thuận lợi, các doanh nghiệp ô tô cần tập trung xây dựng danh tiếng và niềm tin của khách hàng. Ngoài ra, khi doanh nghiệp có danh tiếng xấu, doanh nghiệp sẽ khó cải thiện danh tiếng thông qua các hoạt động CSR.

Cuối cùng, khi khách hàng cảm nhận tốt về các hoạt động CSR, cũng như có niềm tin với doanh nghiệp, khách hàng sẽ hỗ trợ thông qua ý định mua hàng. Vì vậy, các doanh nghiệp cần dành ngân sách để hỗ trợ các hoạt động CSR, đồng thời phải tạo được niềm tin của khách hàng thông qua xây dựng danh tiếng.

\subsection{Hạn chế và hướng nghiên cứu tiếp theo}

Đầu tiên, nghiên cứu xem xét tác động của danh tiếng đến nhận thức CSR tổng hợp của khách hàng. Tuy nhiên, thang đo CSR là thang đo đa chiều, và ảnh hưởng của danh tiếng đến CSR có thể khác nhau ở những khía cạnh khác nhau. Các nghiên cứu tiếp theo cần xem xét mối quan hệ này ở các khía cạnh cụ thể của CSR. Ngoài ra, nghiên cứu này chỉ xem xét một bên liên quan là khách hàng, nghiên cứu tiếp theo có thể mở rộng các bên liên quan khác.

Thứ hai, quan điểm về nhận thức trách nhiệm xã hội doanh nghiệp của khách hàng có nhiều khác biệt tại thị trường Việt Nam. Các nghiên cứu tiếp theo cần phát triển hơn nữa các nhân tố tác động đến nhận thức trách nhiệm xã hội của khách hàng, đồng thời trách nhiệm xã hội doanh nghiệp tác động như thế nào đến thái độ và hành vi của khách hàng.

\section{TÀI LIỆU THAM KHẢO}

[1] Agarwal, J., Osiyevskyy, O., \& Feldman, P. (2015). Corporate reputation measurement: Alternative factor structures, nomological validity, and organizational outcomes. Journal of Business Ethics, 130(2), 485-506.

[2] Ajzen, I., \& Madden, T. (1986). Prediction of goal-directed behavior: Attitudes, intentions, and perceived behavioral control. ournal of experimental social psychology, 22(5), 453-474.

[3] Anderson, E., Fornell, C., \& Mazvancheryl, S. (2004). Customer satisfaction and shareholder value. Journal of marketing, 68(4), 172-185.

[4] Bart, Y., Shankar, V., Sultan, F., \& Urban, G. (2005). Are the drivers and role of online trust the same for all web sites and consumers? A large-scale exploratory empirical study. Journal of marketing, 69(4), 133-152.

[5] Berens, G, \& Van Riel, C. B. (2004). Corporate associations in the academic literature: Three main streams of thought in the reputation measurement literature. Corporate Reputation Review, 7(2), 161-178.

[6] Brown, T. (1998). Corporate associations in Marketing: Antecedents and consequences. Corporate reputation review, 1(3), 215-233.

[7] Carroll, A. (1979). A three-dimensional conceptual model of corporate performance. Academy of management review, 4(4), 497-505.

[8] Fatma, M., \& Rahman, Z. (2015). Consumer perspective on CSR literature review and future research agenda. Management Research Review, 38(2), 195-216.

[9] Fishbein, M., \& Ajzen, I. (1975). Belief. Attitude, Intention and Behavior: An Introduction to Theory and Research Reading. MA: Addison-Wesley.

[10] Fombrun, C. (1996). Reputation. John Wiley \& Sons, Ltd.

[11] Fombrun, C., \& van Riel, C. (1997). The reputation landscape. Corporate Reputation Review, 1(1/2), 5-13. 

NIỀM TIN VÀ Ý ĐỊNH MUA HÀNG CỦA KHÁCH HÀNG TRONG NGÀNH Ô TÔ VIỆT NAM

[12] Freeman, R. E. (1984). Strategic management: a stakeholder approach. Pitman, BostonFreeman RE. Bus Ethics.

[13] Garson, D. (2008). Factor analysis: statnotes. Retrieved March, 22.

[14] Greenwood, M., \& Van Buren III, H. (2010). Trust and stakeholder theory: Trustworthiness in the organisation-stakeholder relationship. Journal of business ethics, 95(3), 425-438.

[15] Ha, H. Y., Janda, S., \& Muthaly, S. K. (2010). A new understanding of satisfaction model in e-re-purchase situation. European Journal of Marketing, 44(7/8), 997-1016.

[16] Helm, S. (2005). Designing a formative measure for corporate reputation. Corporate Reputation Review, 8(2), 95-109.

[17] Hosmer, L. (1995). Trust: The connecting link between organizational theory and philosophical ethics. Academy of management Review, 20(2), 379-403.

[18] Keh, H., \& Xie, Y. (2009). Corporate reputation and customer behavioral intentions: The roles of trust, identification and commitment. Industrial Marketing Management, 38(7), 732-742.

[19] Kim, D. J., Ferrin, D. L., \& Rao, H. R. (2009). Trust and satisfaction, two stepping stones for successful ecommerce relationships: A longitudinal exploration. Information systems research, 20(2), 237-257.

[20] Kramer, R. (1999). Trust and distrust in organizations: Emerging perspectives, enduring questions. Annual review of psychology, 50(1), 569-598.

[21] Lam, S., Shankar, V., Erramilli, M., \& Murthy, B. (2004). Customer Valur, Satisfaction, Loyalty, and Switching Costs: An Lllustration from a Business-to-Business Service Context. Journal of the Academy of Marketing Science, 32(3), 293-311.

[22] Marin, L., Ruiz, S., \& Rubio, A. (2009). The role of identity salience in the effects of corporate social responsibility on consumer behavior. Journal of business ethics, 84(1), 65-78.

[23] McEvily, B., Perrone, V., \& Zaheer, A. (2003). Trust as an organizing principle. Organization science, 14(1), 91-103.

[24] McKnight, D. H., Choudhury, V., \& Kacmar, C. (2002). Developing and validating trust measures for ecommerce: An integrative typology. Information systems research, 13(3), 334-359.

[25] Park, J., Lee, H., \& Kim, C. (2014). Corporate social responsibilities, consumer trust and corporate reputation: South Korean consumers' perspectives. Journal of Business Research, 67(3), 295-302.

[26] Pérez, A. (2015). Corporate reputation and CSR reporting to stakeholders: Gaps in the literature and future lines of research. Corporate Communications: An International Journal, 20(1), 11-29.

[27] Peterson, R. (1994). A meta-analysis of Cronbach's coefficient alpha. Journal of consumer Research , 21(2), 381-403.

[28] Pivato, S., Misani, N., \& Tencati, A. (2008). The impact of corporate social responsibility on consumer trust: the case of organic food. Business ethics: A European review, 17(1), 3-12.

[29] Ponzi, L. J., Fombrun, C. J., \& Gardberg, N. A. (2011). RepTrak ${ }^{\mathrm{TM}}$ pulse: Conceptualizing and validating a short-form measure of corporate reputation. Corporate Reputation Review, Vol.14(Iss.1), 15-35.

[30] Sandhu, H., \& Kapoor , S. (2010). Does it pay to be Socially Responsible? An Empirical Examination of impact of Corporate Social Responsibility on Financial Performance. Global Business Reviews, 11(185), 185208. 

NIỀM TIN VÀ Ý ĐỊNH MUA HÀNG CỦA KHÁCH HÀNG TRONG NGÀNH Ô TÔ VIỆT NAM

[31] Selnes, F., \& Sallis, J. (2003). Promoting relationship learning. Journal of Marketing, 67(3), 80-95.

[32] Sen, S; Bhattacharya, C.B; Korschun, D;. (2006). The Role of Corporate Social Responsibility in Strengthening Multiple Stakeholder Relationships: A Field Experiment. Journal of the Academy of Marketing Science, 34(2), 158-166.

[33] Shim, K., \& Yang, S. U. (2016). The effect of bad reputation: The occurrence of crisis, corporate social responsibility, and perceptions of hypocrisy and attitudes toward a company. Public Relations Review, 42(1), $68-78$.

[34] Singh, J., de los Salmones Sanchez, M., \& del Bosque, I. (2008). Understanding Corporate Social Responsibility and Product Perceptions in Consumer Markets: A Cross-Cultural Evaluation. Journal of Business Ethics, 80(3), 579-611.

[35] Sirdeshmukh, D., Singh, J., \& Sabol, B. (2002). Consumer trust, value, and loyalty in relational exchanges. Journal of marketing, 66(1), 15-37.

[36] Sirohi, N., McLaughlin, E., \& Wittink, D. (1998). A model of consumer perceptions and store loyalty intentions for a supermarket retailer. journal of retailing, 74(2), 223-245.

[37] Van Der Merwe, A. W., \& Puth, G. (2014). Towards a conceptual model of the relationship between corporate trust and corporate reputation. Corporate Reputation Review, 17(2), 138-156.

[38] Walker, K. (2010). A systematic review of the corporate reputation literature: definition, measurement, and theory. Corporate Reputation Review, 12(4), 357-387.

[39] Weiss, A., Anderson, E., \& Maclnnis, D. (1999). Reputation Management as a Motivation for Sales Structure Decisions. Journal of Marketing, 63, 74-89.

[40] Yoon, Y., Gürhan-Canli, Z., \& Schwarz, N. (2006). The effect of corporate social responsibility (CSR) activities on companies with bad reputations. Journal of consumer psychology, 16(4), 377-390.

Ngày nhận bài: 22/07/2017

Ngày chấp nhận đăng: 07/11/2017 